\title{
Quantum wideband traveling-wave analysis of a degenerate parametric amplifier
}

\author{
Carlton M. Caves and David D. Crouch \\ Theoretical Astrophysics 130-33, California Institute of Technology, Pasadena, California 91125
}

Received March 5, 1987; accepted June 5, 1987

\begin{abstract}
We develop a wideband traveling-wave formalism for analyzing quantum mechanically a degenerate parametric amplifier. The formalism is based on spatial differential equations-spatial Langevin equations-that propagate temporal Fourier components of the field operators through the nonlinear medium. In addition to the parametric nonlinearity, the Langevin equations include absorption and associated fluctuations, dispersion (phase mismatching), and pump quantum fluctuations. We analyze the dominant effects of phase mismatching and pump quantum fluctuations on the squeezing produced by a degenerate parametric amplifier.
\end{abstract}

\section{INTRODUCTION}

A degenerate parametric amplifier (DPA) is the prototypic device for generating squeezed-state light. ${ }^{1-3}$ A DPA runs on the nonlinear interaction between a signal field near frequency $\Omega$ and a pump field at frequency $\Omega_{p}=2 \Omega .4$ This parametric interaction has been exploited to generate squeezed-state light ${ }^{5}$-but in an oscillator, rather than an amplifier configuration. In the oscillator configuration the nonlinear medium is enclosed in an optical cavity, in which multiple passes through the medium increase the effective nonlinearity. ${ }^{6-10}$ If one could find materials with larger $\chi^{(2)}$ nonlinearities, however, one might prefer an amplifier configuration because of its intrinsically wider bandwidth.

The conventional approach to quantum problems in nonlinear optics is to specialize to a few interacting modes of the electromagnetic field. All the spatial dependence is contained in the spatial mode functions. The basic equations are temporal differential equations that describe the evolution of the modes. In one realization these equations are temporal operator Langevin equations for the evolution of the creation and annihilation operators of the modes.

This conventional approach is well suited for analyzing a parametric oscillator, in which the appropriate modes are modes of the optical cavity, but it is ill suited for analyzing a DPA, which is a traveling-wave device not easily thought of in terms of a few discrete modes. To analyze a DPA, one would like a set of spatial differential equations for the propagation of the fields through the nonlinear medium. One way to get such spatial differential equations is to take the temporal differential equations for discrete modes interacting parametrically and to replace $t$ with $z / v_{\mathrm{ph}}$, where $v_{\mathrm{ph}}$ is the phase velocity in the medium. ${ }^{11}$ Aside from its questionable validity, this procedure runs into trouble when there is dispersion, and it does not address questions about bandwidths. These problems with the conventional approach have been stressed by Tucker and Walls, ${ }^{12}$ who developed a wave-packet formalism in an attempt to deal with them.

In this paper we model a DPA in a different way. Our approach is patterned after the approach used in classical nonlinear optics, which is formulated in terms of spatial differential equations for coupled Fourier components of the fields. We start with an ideal, lossless, dispersionless medium with a nonlinear susceptibility $\chi^{(2)}$. In such a medium the Heisenberg equations for the field operators are an operator version of the macroscopic Maxwell equations, together with a constitutive relation that includes the nonlinearity. ${ }^{13}$ A temporal Fourier transform then yields spatial differential equations for propagation of the Fourier components of the field operators through the medium. These equations describe a parametric interaction between signal frequencies $\Omega$ $\pm \epsilon$ and the pump at frequency $\Omega_{p}$. Because there is no dispersion, the parametric interaction is perfectly phase matched.

Our next step is to include absorption and dispersion phenomenologically. We replace the actual nonlinear medium by a sequence of slabs of ideal medium separated by beam splitters. Reflection at the beam splitters models a linear loss mechanism, and frequency-dependent phase shifts at the beam splitters introduce dispersion. The final result is a set of spatial propagation equations that include absorption and phase mismatching. These equations might well be called spatial operator Langevin equations for the propagation of the field through the nonlinear medium.

Throughout our analysis we are interested in the dominant effect of quantum fluctuations in the pump field, our goal being to investigate the conditions under which the pump can be treated classically. The dominant effect arises from quantum phase fluctuations in the pump, which feed noise from the amplified signal quadrature into the squeezed signal quadrature. The quantum phase fluctuations in the pump can be viewed as due to vacuum fluctuations at unexcited frequencies near $\Omega_{p}$, which couple to signal frequencies through the parametric nonlinearity. The bandwidth over which such vacuum fluctuations are important, which can be thought of as the pump bandwidth, determines the size of the quantum phase fluctuations in the pump. This pump bandwidth is limited by phase mismatching, which renders frequencies sufficiently far removed from $\Omega_{p}$ effectively uncoupled from the signal frequencies. We evaluate this pump bandwidth within our model. Perhaps surprisingly, 
it is smaller than the bandwidth over which the DPA is phase matched.

In Section 2 we describe our model of a DPA, present a simple heuristic argument for the conditions necessary for a classical pump, and then derive the spatial Langevin equations for the model. In Section 3 we use the spatial Langevin equations to investigate the effects of phase mismatching and pump quantum fluctuations on the squeezing spectrum.

\section{MODEL FOR THE DEGENERATE PARAMETRIC AMPLIFIER}

\section{A. Description of Model}

Consider a nonlinear medium of length $L$, which lies between $z=0$ and $z=L$. The nonlinearity is described by a nonlinear susceptibility $\chi^{(2)}$. Propagating through the medium in the $+z$ direction is a strong pump wave at frequency $\Omega_{p}$ and a signal wave at frequencies near the degeneracy frequency $\Omega=\Omega_{p} / 2$. The parametric nonlinearity couples the pump to signal frequencies $\Omega \pm \epsilon$. We idealize all the waves as plane waves with a single polarization in which the electric (magnetic) field lies along the $x$ axis ( $y$ axis). Specializing to a single polarization ignores the details of how phase matching is achieved in many real DPA's, but these details are not important for our quantum analysis. Specializing to plane waves ignores the transverse structure of the waves, which we, nonetheless, take into account crudely by introducing an effective cross-sectional area $\sigma$ for the waves.

We assume for simplicity that the index of refraction for frequiencies near $\Omega_{p}$ is uniform with value $n_{p}=n_{0}$; hence the pump wave number is $K_{p}=\Omega_{p} n_{0} / c$. The index of refraction for frequencies $\omega=\Omega \pm \epsilon$ near $\Omega$ is allowed to be dispersive with value $n(\omega)=n_{0}+\Delta n(\omega)$; the corresponding wave number is denoted $k(\omega)=\omega n(\omega) / c$. We assume perfect phase matching at degeneracy, i.e., $\Delta n(\Omega)=0$, so that the wave number at degeneracy is $K=\Omega n_{0} / c=K_{p} / 2$.

We assume, for convenience, that the medium is lossless for frequencies near $\Omega_{p}$, but we allow for absorption at frequencies $\omega$ near $\Omega$. The absorption is characterized by an absorption coefficient $\gamma(\omega)$, which gives the loss per unit length in photon units.

The pump's magnetic field has complex amplitude $i A_{p} e^{i \phi_{p}}$ $=i A_{p} e^{2 i \phi}$, where $A_{p}$ is the pump amplitude and $\phi_{p}=2 \phi$ is the pump phase. The corresponding pump power is $P_{p}=(c \sigma)$ $\left.8 \pi n_{0}\right) A_{p}{ }^{2}$.

It is useful to introduce a dimensionless measure of the nonlinearity,

$$
\alpha_{0} \equiv \frac{2 \pi \chi^{(2)} A_{p}}{n_{0}{ }^{2}}=2 \pi \frac{\chi^{(2)}}{n_{0}^{3 / 2}}\left(\frac{8 \pi P_{p}}{c \sigma}\right)^{1 / 2},
$$

in terms of which the nonlinear gain coefficient of the medium is

$$
g_{0} \equiv \alpha_{0}(\Omega / c)=\alpha_{0}\left(K / n_{0}\right) .
$$

There are three important spatial rates in our model: (i) the rate of accumulation of phase $K$, (ii) the nonlinear gain coefficient $g_{0}$, and (iii) the absorption coefficient $\gamma$. The fundamental assumption of our analysis-just as it is the fundamental assumption of a classical analysis of a DPA is that these spatial rates satisfy

$$
g_{0}, \gamma \ll K .
$$

That $g_{0} \ll K$ is equivalent to saying that the dimensionless nonlinearity $\alpha_{0} \ll 1$.

The nonlinear gain is effective only over the bandwidth for which the medium is phase matched. The extent of phase mismatching at frequencies $\Omega \pm \epsilon$ is characterized by

$$
\begin{aligned}
\Delta k(\epsilon) & \equiv K_{p}-k(\Omega+\epsilon)-k(\Omega-\epsilon) \\
& =-\frac{(\Omega+\epsilon) \Delta n(\Omega+\epsilon)}{c}-\frac{(\Omega-\epsilon) \Delta n(\Omega-\epsilon)}{c} .
\end{aligned}
$$

The index of refraction varies only a small amount over the phase-matched bandwidth, so we can expand it as

$$
\Delta n(\Omega \pm \epsilon)= \pm n^{\prime} \epsilon+1 / 2 n^{\prime \prime} \epsilon^{2},
$$

where the derivatives of $n(\omega)$ are evaluated at $\Omega$. One then finds that

$$
\Delta k(\epsilon)=-p \epsilon^{2} / \Omega c, \quad p \equiv 2 \Omega n^{\prime}+\Omega^{2} n^{\prime \prime},
$$

where $p$ is a dimensionless factor for which a typical value might be $|p| \sim 0.1$. One can now introduce frequencies $\epsilon_{1}$ and $\epsilon_{2}$ at which the medium begins to be badly mismatched:

$$
\begin{aligned}
\left|\Delta k\left(\epsilon_{1}\right) L\right| & =1 \Rightarrow \epsilon_{1}=|p|^{-1 / 2}(\Omega c / L)^{1 / 2}, \\
\left|\Delta k\left(\epsilon_{2}\right) / 2 g_{0}\right| & =1 \Rightarrow \epsilon_{2}=|p|^{-1 / 2}\left(2 \Omega c g_{0}\right)^{1 / 2} .
\end{aligned}
$$

The bandwidth $\Delta / 2 \pi$ over which the DPA is phase matched can be defined as

$$
\Delta / 2 \pi \equiv \pi^{-1} \min \left(\epsilon_{1}, \epsilon_{2}\right) \ll \Omega / 2 \pi .
$$

\section{B. Conditions for a Classical Pump}

The pump wave is, of course, not completely classical. Its monochromatic excitation at frequency $\Omega_{p}$ is inevitably accompanied by quantum fluctuations, which lead to fluctuations in the pump amplitude and phase. The limit in which the quantum fluctuations can be totally ignored and the pump is strictly classical is not just the limit of a very strong pump; rather, it is that the pump amplitude $A_{p} \rightarrow \infty$, while the nonlinear susceptibility $\chi^{(2)} \rightarrow 0$, in such a way that the dimensionless nonlinearity $\alpha_{0} \propto \chi^{(2)} A_{p}$ (or the nonlinear gain $g_{0}$ ) is held constant. Knowing this limit, however, does not tell one whether the pump in a given DPA can be treated classically to a good approximation. Indeed, the important practical question concerns a given nonlinear medium with a fixed value of $\chi^{(2)}$-not a fixed value of $\alpha_{0}$. One would like to know, given $\chi^{(2)}$, the range of pump powers $P_{p}$ for which the pump is approximately classical.

There is a simple heuristic argument ${ }^{14}$ for the dominant effect of pump quantum fluctuations. If the DPA were powered by a classical pump, it would produce ideal squeezed light at phase-matched signal frequencies. Such ideal squeezed light can be represented in a complex-amplitude diagram by an ellipse ${ }^{15,16}$ with radius $e^{-g_{0} L}$ for the squeezed quadrature and radius $e^{g_{0} L}$ for the amplified quadrature (ellipse with solid lines in Fig. 1). The orientation of the ellipse is determined by the phase of the pump; in Fig. 1 the pump phase is chosen so that the ellipse with solid lines is oriented along the real and imaginary axes. 


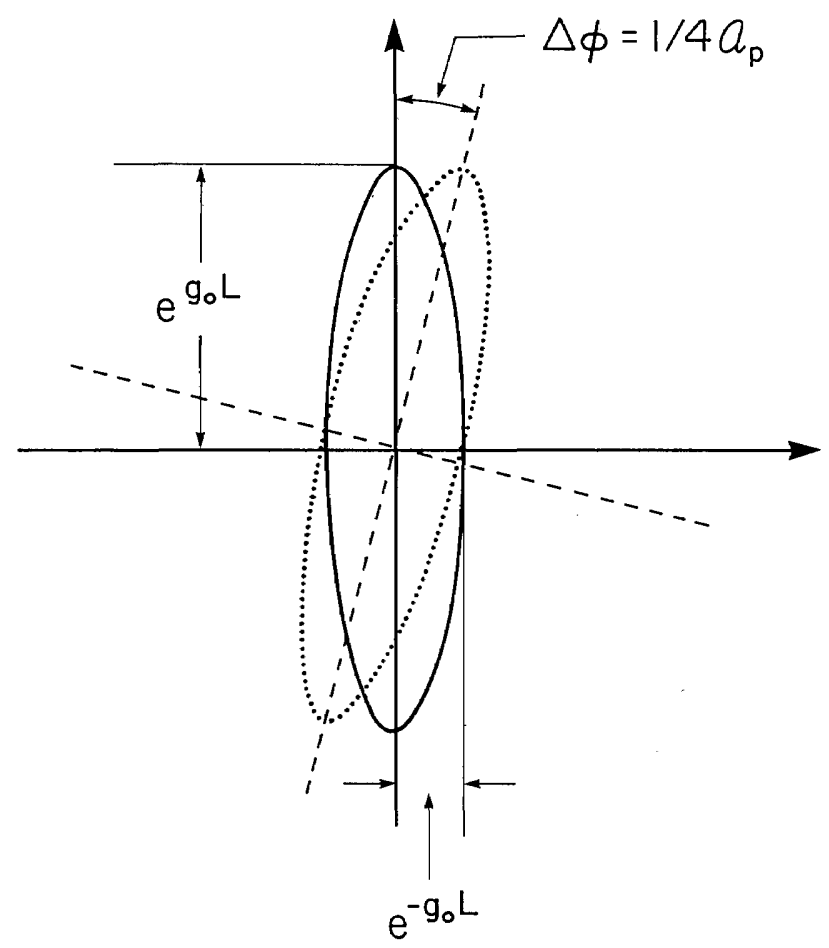

Fig. 1. Effect of pump quantum fluctuations on squeezing. Ideal squeezing is represented by the ellipse with solid lines. Pump phase fluctuations cause the orientation of the ellipse to fluctuate through a characteristic angle $\Delta \phi=1 / 4 \mathcal{A}_{p}$, as indicated schematically by the dotted ellipse. These fluctuations feed noise from the amplified signal quadrature into the squeezed signal quadrature.

The dominant effect of pump quantum fluctuations arises from the phase fluctuations, which have characteristic size $\Delta \phi_{p}=1 / 2 N_{p}{ }^{1 / 2}=1 / 2 \mathcal{A}_{p}$. Here $N_{p}$ is the number of pump photons, and $\mathcal{A}_{p}$ is a dimensionless pump amplitude in photon units. The pump phase fluctuations cause the orientation of the ellipse in Fig. 1 to fluctuate, as indicated schematically by the dotted ellipse. The characteristic angle through which the ellipse turns is $\Delta \phi=\Delta \phi_{p} / 2=1 / 4 \mathcal{A}_{p}$. These orientation fluctuations feed noise from the amplified signal quadrature into the squeezed signal quadrature, thereby degrading the squeezing. The characteristic size of the noise added to the squeezed quadrature is $\Delta \phi e^{g_{0} L}$. Thus the condition for a classical pump is

$$
\Delta \phi e^{g_{0} L}=e^{g_{0} L} / 4 \mathcal{A}_{p} \ll e^{-g_{0} L} \Longrightarrow \mathcal{A}_{p} \gg 1 / 4 e^{2 g_{0} L}=1 / 4 e^{2 \alpha_{0}(\Omega L / c)} .
$$

One sees in this condition the limit for a strictly classical pump: $\alpha_{0}=$ constant, $\mathcal{A}_{p} \rightarrow \infty$.

Missing from the preceding argument is any hint of how to relate the physical pump amplitude $A_{p}$ to the dimensionless amplitude $\mathcal{A}_{p}$. That relation requires identifying an appropriate bandwidth. The pump quantum fluctuations can be regarded as arising from vacuum fluctuations in unexcited frequencies near $\Omega_{p}$, which are coupled to signal frequencies by the parametric nonlinearity. The size of the phase fluctuations-and, hence, the effective number of pump photons-depends on the bandwidth of nearby frequencies that must be considered. Clearly this pump bandwidth $\Delta_{p} / 2 \pi$ is limited by phase mismatching, which means that frequencies sufficiently far removed from $\Omega_{p}$ are not effectively coupled to frequencies in the signal field.
Given a pump bandwidth $\Delta_{p} / 2 \pi$, one can identify the number of pump photons as

$$
N_{p} \equiv \frac{P_{p}}{\hbar \Omega_{p} \Delta_{p} / 2 \pi}=\frac{c \sigma}{4 n_{0} \hbar \Omega_{p} \Delta_{p}} A_{p}^{2}=\left(\frac{A_{p}}{A_{\text {vac }}}\right)^{2} \equiv \mathcal{A}_{p}^{2} .
$$

Here

$$
A_{\mathrm{vac}} \equiv\left(4 n_{0} \hbar \Omega_{p} \Delta_{p} / c \sigma\right)^{1 / 2}
$$

is an effective amplitude for the pump quantum fluctuations; it corresponds to a vacuum power $P_{\text {vac }}=\left(c \sigma / 8 \pi n_{0}\right)$ $\times A_{\mathrm{vac}^{2}}{ }^{2}=\hbar \Omega_{p} \Delta_{\mathrm{p}} / 2 \pi$. Notice that the variance in the pump phase is

$$
\left(\Delta \phi_{p}\right)^{2}=1 /{ }_{4} N_{p}=\left(\hbar \Omega_{p} / 4 P_{p}\right)\left(\Delta_{p} / 2 \pi\right) .
$$

It is useful to introduce a dimensionless nonlinear susceptibility

$$
\alpha_{\mathrm{vac}} \equiv \frac{\alpha_{0}}{\mathcal{A}_{p}}=\frac{2 \pi \chi^{(2)} A_{\mathrm{vac}}}{n_{0}{ }^{2}},
$$

which measures the intrinsic nonlinearity of the medium in units of the pump vacuum amplitude.

Now write condition (2.9) for a classical pump as

$$
\mathcal{A}_{p} \gg 1 / 4 \exp \left[2 \alpha_{\text {vac }}(\Omega L / c) \mathcal{A}_{p}\right]
$$

For a given nonlinear medium with a fixed value of $\alpha_{\text {vac }}(\Omega L / c)$, there is a restricted range of pump amplitudes for which the pump is approximately classical. The upper end of the range is determined by the solution of

$$
\mathcal{A}_{\text {max }}=1 / 4 \exp \left[2 \alpha_{\text {vac }}(\Omega L / c) \mathcal{A}_{\text {max }}\right] ;
$$

to be approximately classical, the pump must have dimensionless amplitude $\mathcal{A}_{p}$ much bigger than 1 but somewhat smaller than $\mathcal{A}_{\text {max }}$. Rewritten in terms of physical parameters, the condition for a classical pump becomes

$$
P_{p} \gg \frac{1}{16} \hbar \Omega_{p} \frac{\Delta_{p}}{2 \pi} \exp \left[8 \pi \frac{\chi^{(2)}}{n_{0}^{3 / 2}}\left(\frac{\Omega L}{c}\right)\left(\frac{8 \pi P_{p}}{c \sigma}\right)^{1 / 2}\right] .
$$

These considerations hinge on knowing the pump bandwidth $\Delta_{p} / 2 \pi$. One's first guess might be that $\Delta_{p} / 2 \pi$ is about the same size as the phase-matched bandwidth $\Delta / 2 \pi$, but our detailed calculation in Subsection 3.D confirms the preceding argument and shows that, within our model,

$$
\Delta_{p} / 2 \pi=c g_{0} / \Omega\left|n^{\prime}\right|
$$

which is typically smaller than $\Delta / 2 \pi$.

Previous analyses of pump fluctuations in a DPA have idealized to a few discrete modes; thus they do not address bandwidth questions. Wódkiewicz and Zubairy ${ }^{17}$ specialized to a single-mode pump and a single-mode signal, and they analyzed classical fluctuations in the pump amplitude and phase. Their result is consistent with the above argument, with $\Delta \phi_{p}$ given by classical phase diffusion instead of quantum fluctuations. Hillery and Zubairy ${ }^{14}$ considered a single-mode pump and a single-mode signal, and they evaluated the effect of pump quantum fluctuations by using a path-integral analysis. Their result is consistent with the preceding argument. Scharf and Walls ${ }^{18}$ specialized to a single-mode pump and a two-mode signal (signal and idler modes), and they did a detailed asymptotic analysis of pump 
quantum fluctuations. In their analysis the dominant effect of pump quantum fluctuations is an error term $e^{3 g_{0} L} / \sqrt{480} \mathcal{A}_{p}$ (in our notation), a bigger effect than the dominant effect $e^{g_{0} L} / 4 \mathcal{A}_{p}$ suggested by the above argument. If the ScharfWalls result is correct, then pump quantum fluctuations are more serious than our analysis indicates.

\section{Spatial Langevin Equations}

We are primarily interested in the behavior of the signal field. Propagating toward the nonlinear medium from the vacuum region $z<0$ is the input signal field, a free field whose positive-frequency field operators can be written as

$$
\begin{aligned}
B_{\mathrm{in}}{ }^{(+)}= & D_{\mathrm{in}}{ }^{(+)}=E_{\mathrm{in}}{ }^{(+)}=\int_{\mathcal{B}_{s}} \frac{\mathrm{d} \omega}{2 \pi}\left(\frac{2 \pi \hbar \omega}{c \sigma}\right)^{1 / 2} \\
& \times a_{\mathrm{in}}(\omega) \exp [i \omega(z / c-t)], \quad z \leqq 0,
\end{aligned}
$$

where the integral runs over a bandwidth $\mathscr{B}_{s}$ about $\Omega$, which contains all relevant signal frequencies. The operators $a_{\text {in }}(\omega)$ and $a_{\text {in }}^{\dagger}(\omega)$ are annihilation and creation operators for the input signal field; they satisfy continuum commutation relations

$$
\left[a_{\text {in }}(\omega), a_{\text {in }}^{\dagger}\left(\omega^{\prime}\right)\right]=2 \pi \delta\left(\omega-\omega^{\prime}\right)
$$

The total energy (power integrated over all time) transported by the input signal field through a surface $z=$ constant is

$$
\int_{\mathcal{B}_{s}} \frac{\mathrm{d} \omega}{2 \pi} \hbar \omega a_{\mathrm{in}}^{\dagger}(\omega) a_{\mathrm{in}}(\omega) .
$$

Similar considerations apply to the output signal field, which propagates away from the nonlinear medium into the vacuum region $z>L$. We denote it in the same way as the input signal field, but with the designation "in" replaced by "out."

Inside the nonlinear medium we write the signal field in terms of a temporal Fourier expansion. The positive-frequency part of the magnetic-field operator is given by

$$
\begin{aligned}
B_{s}{ }^{(+)} & =\int_{\mathcal{B}_{s}} \frac{\mathrm{d} \omega}{2 \pi} B_{s}(\omega, z) \exp [i(k z-\omega t)], \\
k & =\omega n(\omega) / c, \quad 0 \leqq z \leqq L, \\
B_{s}(\omega, z) & =\left[\frac{c}{n(\omega) v_{g}(\omega)}\right]^{1 / 2}\left[\frac{2 \pi n(\omega) \hbar \omega}{c \sigma}\right]^{1 / 2} a_{s}(\omega, z) .
\end{aligned}
$$

Here $v_{g}(\omega) \equiv(\mathrm{d} k / \mathrm{d} \omega)^{-1}$ is the group velocity in the medium.

If there were no nonlinearity, the Fourier components $B_{s}(\omega, z)$ would have no $z$ dependence, and the displacementfield operator $D_{s}{ }^{(+)}$and the electric-field operator $E_{s}{ }^{(+)}$ would have Fourier expansions similar to Eq. (2.20a), with $D_{s}(\omega)=[n(\omega)]^{2} E_{s}(\omega)=n(\omega) B_{s}(\omega)$. In addition, the total energy transported by the signal field through a surface $z=$ constant would be

$$
\int_{\mathcal{B}_{s}} \frac{\mathrm{d} \omega}{2 \pi} \hbar \omega a_{s}^{\dagger}(\omega) a_{s}(\omega)
$$

[the group-velocity factor in Eq. (2.20b) is included to ensure this form]. Since the nonlinearity is small, we ignore the energy stored in the nonlinear polarization; hence we can write the total energy transported by the signal field through a surface $z=$ constant in the nonlinear medium as

$$
\int_{\mathcal{B}_{s}} \frac{\mathrm{d} \omega}{2 \pi} \hbar \omega a_{s}^{\dagger}(\omega, z) a_{s}(\omega, z) .
$$

Thus the operators $a_{s}(\omega, z)$ are Fourier components normalized to be in photon units.

These considerations show that, if there are no reflections at the input and output surfaces (perfect antireflection coatings), then appropriate boundary conditions are

$$
a_{s}(\omega, 0)=a_{\text {in }}(\omega), \quad a_{\text {out }}(\omega)=a_{s}(\omega, L) .
$$

A priori one does not know the commutators of the Fourier components $a_{s}(\omega, z)$, because knowing them would require knowing the nonequal-time field commutators. Nonetheless, in this simple case of plane waves propagating in one direction with no reflections, the above boundary conditions specify the commutators for $a_{s}(\omega, 0)$ and $a_{s}^{\dagger}\left(\omega^{\prime}, 0\right)$ and also the commutators for $a_{s}(\omega, L)$ and $a_{s}^{\dagger}\left(\omega^{\prime}, L\right)$. Further, since the output boundary could be put at any value of $z$, one in fact knows the equal-position commutators for any value of $z$ :

$$
\left[a_{s}(\omega, z), a_{s}^{\dagger}\left(\omega^{\prime}, z\right)\right]=2 \pi \delta\left(\omega-\omega^{\prime}\right) .
$$

Besides the signal field, one also needs the pump field. Inside the medium we expand the pump's magnetic-field operator as

$$
\begin{gathered}
B_{p}^{(+)}=\int_{\mathcal{B}_{p}} \frac{\mathrm{d} \omega}{2 \pi} B_{p}(\omega, z) \exp \left[i\left(k_{0} z-\omega t\right)\right] \\
k_{0}=\omega n_{0} / c, \quad 0 \leqq z \leqq L, \\
B_{p}(\omega, z)=\frac{1}{2} i A_{p} e^{i \phi_{p}} 2 \pi \delta\left(\omega-\Omega_{p}\right)+\left(\frac{2 \pi n_{0} \hbar \omega}{c \sigma}\right)^{1 / 2} a_{p}(\omega, z) .
\end{gathered}
$$

Here the integral runs over a bandwidth $\mathcal{B}_{p}$ about $\Omega_{p}$, which contains all relevant pump frequencies. The first term in $B_{p}(\omega, z)$ is the strong mean pump field, and the second term represents fluctuations about the mean. Considerations identical to those for the signal field show that $a_{p}(\omega, 0)$ and $a_{p}(\omega, L)$ are input and output annihilation operators at frequency $\omega$. Throughout our analysis we assume that, aside from the strong excitation at frequency $\Omega_{p}$, the input pump field is in the vacuum state.

We would like to include in our description absorption and dispersion in the signal field, but there is a difficulty in doing so. The equations that we use to describe the nonlinearity are an operator version of the macroscopic Maxwell equations, which are the Heisenberg equations derived from an appropriate Hamiltonian. It is difficult to include losses and dispersion in such a Hamiltonian formalism. ${ }^{13}$ Therefore we separate the losses and dispersion from the nonlinearity by using a trick (Fig. 2): Suppose that we have managed to propagate the signal and pump fields to position $z$ in the medium and we wish to propagate them a further small distance $\Delta z$. We replace the actual medium between $z$ and $z$ $+\Delta z$ by a beam splitter followed by a slab of $i d e a l$ nonlinear medium, which has no absorption and no dispersion. The reflectivity of the beam splitter accounts for losses, and frequency-dependent phase shifts at the beam splitter introduce dispersion. The ideal nonlinear medium has uniform index of refraction $n_{0}$ and nonlinear susceptibility $\chi^{(2)}$. 

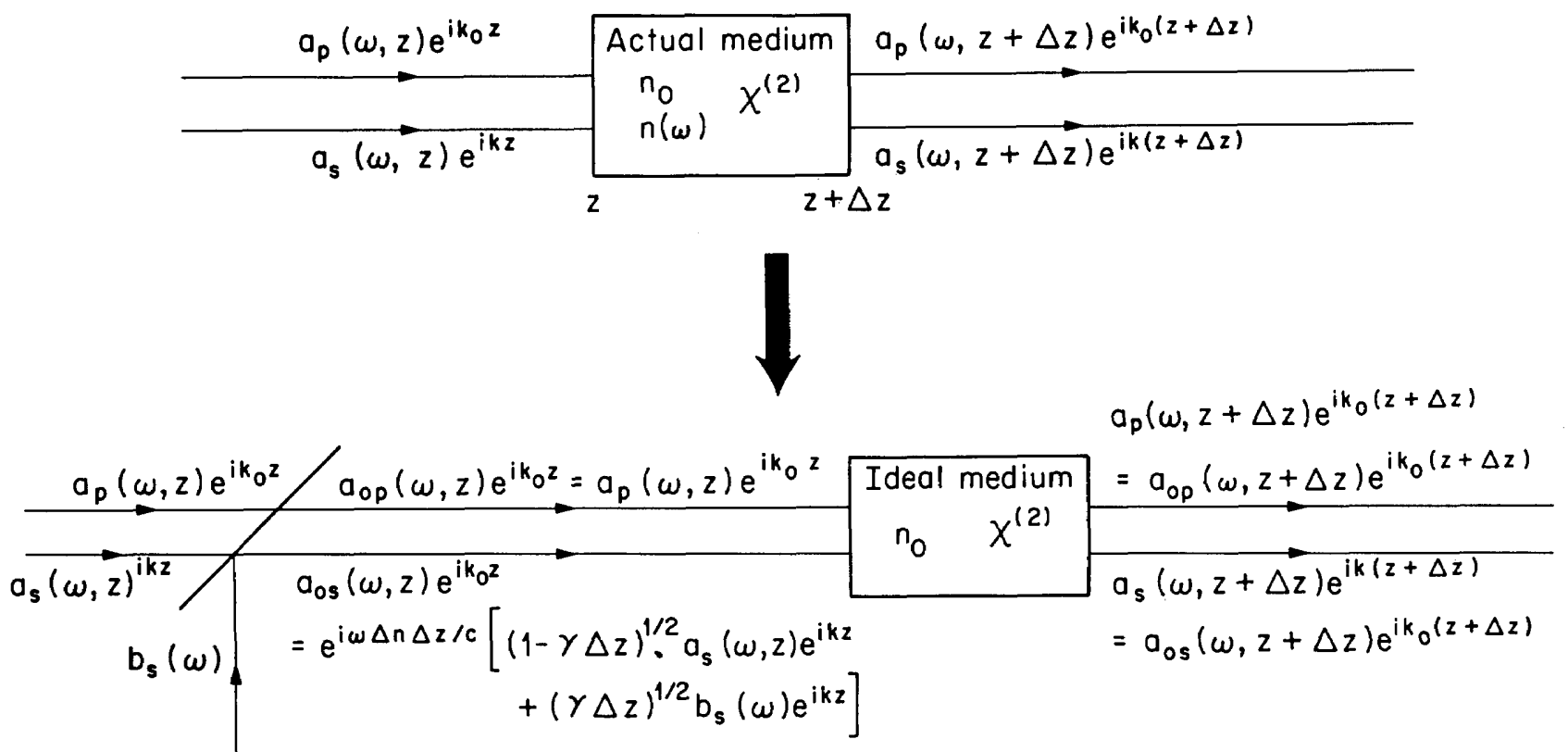

Fig. 2. Trick for introducing absorption and dispersion (phase mismatching). The actual nonlinear medium between $z$ and $z+\Delta z$ is replaced by a slab of ideal (lossless, dispersionless) nonlinear medium preceded by a beam splitter. Reflection at the beam splitter accounts for losses, and frequency-dependent phase shifts at the beam splitter introduce dispersion.

The problem that must be solved is to relate the fields entering the actual medium at $z+\Delta z$, i.e., the Fourier components $a_{s}(\omega, z+\Delta z) \exp [i k(z+\Delta z)]$ and $a_{p}(\omega, z+\Delta z)$ $\times \exp \left[i k_{0}(z+\Delta z)\right]$, to the fields leaving the actual medium at $z$, i.e., the Fourier components $a_{s}(\omega, z) e^{i k z}$ and $a_{p}(\omega, z) e^{i k_{0} z}$ (Fig. 2). To do this, we need to know how to propagate the fields through the beam splitter and the ideal nonlinear medium.

As a first step, we need to describe the fields within the ideal nonlinear medium. We denote these fields by a subscript 0 , and we write each field operator as a sum of a signal field and a pump field. For example, the positive-frequency part of the magnetic-field operator within the ideal nonlinear medium is

$$
B_{0}{ }^{(+)}=B_{0 s}{ }^{(+)}+B_{0 p}{ }^{(+)} \text {. }
$$

The signal and pump fields are written in terms of temporal Fourier transforms:

$$
\begin{aligned}
B_{0 s}{ }^{(+)}= & \int_{\mathcal{B}_{s}} \frac{\mathrm{d} \omega}{2 \pi} B_{0 s}(\omega, \xi) \exp \left[i\left(k_{0} \xi-\omega t\right)\right] \\
k_{0}=\omega n_{0} / c & \\
B_{0 s}(\omega, \xi)= & \left(\frac{2 \pi n_{0} \hbar \omega}{c \sigma}\right)^{1 / 2} a_{0 s}(\omega, \xi) \\
B_{0 p}{ }^{(+)}= & \int_{\mathcal{B}_{p}} \frac{\mathrm{d} \omega}{2 \pi} B_{0 p}(\omega, \xi) \exp \left[i\left(k_{0} \xi-\omega t\right)\right] \\
B_{0 p}(\omega, \xi)= & \frac{1}{2} i A_{p} e^{i \phi_{p} 2 \pi \delta\left(\omega-\Omega_{p}\right)} \\
& +\left(\frac{2 \pi n_{0} \hbar \omega}{c \sigma}\right)^{1 / 2} a_{0 p}(\omega, \xi)
\end{aligned}
$$

$(z \leqq \xi \leqq z+\Delta z)$. Just as before, the operators $a_{0 s}(\omega, \xi)$ and $a_{0 p}(\omega, \xi)$ give the energy transported in photon units, so it is appropriate to impose boundary conditions in terms of them.

The beam splitter transforms the fields that leave the actual medium at $z$ before they reach the slab of ideal medium. Since the beam splitter has no effect on the pump field, the appropriate transformation at pump frequencies is

$$
a_{0 p}(\omega, z)=a_{p}(\omega, z)
$$

At signal frequencies the beam splitter has frequencydependent reflectivity $\gamma(\omega) \Delta z$, which thus becomes the loss in photon units at frequency $\omega$ within the slab $\Delta z$. In other words, $\gamma(\omega)$ is the absorption coefficient (loss per unit length) of the actual medium. To conserve energy (or to preserve unitarity), the beam splitter must have a second input port, into which propagates a free field with annihilation operators $b_{s}(\omega)$, satisfying continuum commutation relations

$$
\left[b_{s}(\omega), b_{s}^{\dagger}\left(\omega^{\prime}\right)\right]=2 \pi \delta\left(\omega-\omega^{\prime}\right)
$$

This auxiliary signal field accounts for fluctuations associated with absorption; it is assumed to be in the vacuum state. The transformation law for the beam splitter at signal frequencies is

$$
\begin{aligned}
a_{0 s}(\omega, z) e^{i k_{0} z}= & \exp [i \omega \Delta n(\omega) \Delta z / c]\left\{[1-\gamma(\omega) \Delta z]^{1 / 2} a_{s}(\omega, z) e^{i k z}\right. \\
& \left.+[\gamma(\omega) \Delta z]^{1 / 2} b_{s}(\omega) e^{i k z}\right\}
\end{aligned}
$$

The phase factors $e^{i k z}$ and $e^{i k_{0} z}$ are included so as to match the phase of the field leaving the actual medium to the phase of the field entering the ideal medium. The frequencydependent phase shift at the beam splitter, $\omega \Delta n(\omega) \Delta z / c$, where $\Delta n(\omega) \equiv n(\omega)-n_{0}$, is simply the additional phase shift 
required to account for dispersion in the actual medium within the slab $\Delta z$.

It is useful in what follows to write $b_{s}(\omega)$ as an integral over a continuum of contributions within the slab $\Delta z$ :

$$
b_{s}(\omega)=(\Delta z)^{-1 / 2} \int_{z}^{2+\Delta z} \mathrm{~d} \xi b_{s}(\omega, \xi)
$$

The operators $b_{s}(\omega, \xi)$ obey the commutation relations

$$
\left[b_{s}(\omega, \xi), b_{s}^{\dagger}\left(\omega^{\prime}, \xi^{\prime}\right)\right]=2 \pi \delta\left(\omega-\omega^{\prime}\right) \delta\left(\xi-\xi^{\prime}\right) .
$$

At the far end of the slab, the appropriate boundary conditions to get back into the actual medium are

$$
\begin{aligned}
& a_{p}(\omega, z+\Delta z)=a_{0 p}(\omega, z+\Delta z) \\
& a_{s}(\omega, z+\Delta z) \exp [i k(z+\Delta z)]= a_{0 s}(\omega, z+\Delta z) \\
& \times \exp \left[i k_{0}(z+\Delta z)\right] .
\end{aligned}
$$

Just as above, the phase factors in Eq. (2.33) match the phase of the field leaving the ideal medium to the phase of the field entering the actual medium.

What remains now is to propagate the field through the slab of ideal nonlinear medium. If we describe the nonlinearity by a susceptibility $\chi^{(2)}$, then the Heisenberg equations for a lossless, dispersionless nonlinear medium are simply an operator version of the macroscopic Maxwell equations, supplemented by a constitutive relation. ${ }^{13}$ The two important Maxwell equations are $c^{-1} \partial D_{0 q}{ }^{(+)} / \partial t=-\partial B_{0 q}{ }^{(+)} / \partial \xi$ and $\partial E_{0 q}{ }^{(+)} / \partial \xi=-c^{-1} \partial B_{0 q}{ }^{(+)} / \partial t$, where $q$ can stand for either $s$ or $p$. We choose to write a constitutive relation for the electric field in terms of the displacement field ${ }^{13}$ rather than the usual relation for the displacement field in terms of the electric field. Thus we use a nonlinear susceptibility $\eta^{(2)}=$ $n_{0}{ }^{-6} \chi^{(2)}$. Decomposed in terms of signal and pump fields, the constitutive relation becomes

$$
\begin{aligned}
& E_{0 s}{ }^{(+)}=n_{0}{ }^{-2} D_{0 s}{ }^{(+)}-8 \pi \eta^{(2)} D_{0 p}{ }^{(+)} D_{0 s}{ }^{(-)}, \\
& E_{0 p}{ }^{(+)}=n_{0}{ }^{-2} D_{0 p}{ }^{(+)}-4 \pi \eta^{(2)}\left[D_{0 s}{ }^{(+)}\right]^{2} .
\end{aligned}
$$

By plugging the Fourier expansions [Eqs. (2.25) and (2.26)] into the Maxwell equations and the constitutive relation and by keeping only the highest-order terms in $\alpha_{0} \ll 1$ and $\alpha_{0} / \mathcal{A}_{p} \ll \alpha_{0}$, we find the following two spatial propagation equations:

(i) a signal equation,

$$
\begin{aligned}
\frac{\mathrm{d} a_{0 s}(\omega, \xi)}{\mathrm{d} \xi}= & -g_{0}\left[\frac{\omega\left(\Omega_{p}-\omega\right)}{\Omega^{2}}\right]^{1 / 2} e^{2 i \phi} a_{0 s}^{\dagger}\left(\Omega_{p}-\omega, \xi\right) \\
& +i \frac{g_{0}}{\mathcal{A}_{p}} \int_{\mathcal{B}_{p}} \frac{\mathrm{d} \omega^{\prime}}{2 \pi}\left[\frac{\omega \omega^{\prime}\left(\omega^{\prime}-\omega\right)}{\Omega^{2} \Omega_{p}}\right]^{1 / 2} \\
& \times \frac{a_{0 p}\left(\omega^{\prime}, \xi\right)}{\left(\Delta_{p} / 2 \pi\right)^{1 / 2}} a_{0 s}^{\dagger}{ }^{\dagger}\left(\omega^{\prime}-\omega, \xi\right)
\end{aligned}
$$

(ii) a pump equation,

$$
\begin{aligned}
\frac{\mathrm{d} a_{0 p}(\omega, \xi)}{\mathrm{d} \xi}= & \frac{i}{2} \frac{g_{0}}{\mathcal{A}_{p}} \int_{\mathcal{B}_{s}} \frac{\mathrm{d} \omega^{\prime}}{2 \pi}\left[\frac{\omega \omega^{\prime}\left(\omega-\omega^{\prime}\right)}{\Omega^{2} \Omega_{p}}\right]^{1 / 2} \\
& \times \frac{a_{0 s}\left(\omega^{\prime}, \xi\right) a_{0 s}\left(\omega-\omega^{\prime}, \xi\right)}{\left(\Delta_{p} / 2 \pi\right)^{1 / 2}}
\end{aligned}
$$

The first term in the signal equation is the primary effect of the parametric nonlinearity. It is the standard nonlinear coupling, mediated by the pump at frequency $\Omega_{p}$, between signal frequencies $\omega=\Omega+\epsilon$ and $\Omega_{p}-\omega=\Omega-\epsilon$. The second term is an integral over equivalent couplings mediated by initially unexcited pump frequencies $\omega^{\prime}$ within the bandwidth $\mathscr{B}_{p}$; it includes the effects of pump quantum fluctuations. The pump equation describes an integral over nonlinear couplings between a pump frequency $\omega$ and signal frequencies $\omega^{\prime}$ and $\omega-\omega^{\prime}$; it includes, for example, the effects of pump depletion.

Equations (2.35) are the desired equations for propagation through the slab of ideal nonlinear medium. If we assume that the slab is sufficiently thin that $g_{0} \Delta z \ll 1$, then we can approximate the solutions of Eqs. (2.35) as

$$
\begin{aligned}
& a_{0 s}(\omega, z+\Delta z)=a_{0 s}(\omega, z)+\left.\frac{\mathrm{d} a_{0 s}(\omega, \xi)}{\mathrm{d} \xi}\right|_{\xi=z} \Delta z, \\
& a_{0 p}(\omega, z+\Delta z)=a_{0 p}(\omega, z)+\left.\frac{\mathrm{d} a_{0 p}(\omega, \xi)}{\mathrm{d} \xi}\right|_{\xi=z} \Delta z .
\end{aligned}
$$

If we further assume that $\gamma(\omega) \Delta z \ll 1$ and $\omega \Delta n(\omega) \Delta z / c \ll 1$ and linearize in these quantities, we can combine Eqs. (2.27), (2.29), (2.32), (2.33), (2.35), and (2.36) to relate the fields entering the actual medium at $z+\Delta z$ to the fields leaving the actual medium at $z$. By taking the limit $\Delta z \rightarrow 0$ and simultaneously introducing the operators $b_{s}(\omega, \xi)$ of Eq. (2.30), we can rewrite these relations as the following two spatial differential equations:

(i) a signal equation,

$$
\begin{aligned}
\frac{\mathrm{d} a_{s}(\omega, z)}{\mathrm{d} z}= & -\frac{1}{2} \gamma(\omega) a_{s}(\omega, z) \\
& -g_{0}\left[\frac{\omega\left(\Omega_{p}-\omega\right)}{\Omega^{2}}\right]^{1 / 2} e^{2 i \phi} \exp \left[i \Delta K\left(\Omega_{p}, \omega\right) z\right] \\
& \times a_{s}^{\dagger}\left(\Omega_{p}-\omega, z\right)+[\gamma(\omega)]^{1 / 2} b_{s}(\omega, z) \\
& +i \frac{g_{0}}{\mathcal{A}_{p}} \int_{\mathcal{B}_{p}} \frac{\mathrm{d} \omega^{\prime}}{2 \pi}\left[\frac{\omega \omega^{\prime}\left(\omega^{\prime}-\omega\right)}{\Omega^{2} \Omega_{p}}\right]^{1 / 2} \exp \left[i \Delta K\left(\omega^{\prime}, \omega\right) z\right] \\
& \times \frac{a_{p}\left(\omega^{\prime}, z\right)}{\left(\Delta_{p} / 2 \pi\right)^{1 / 2}} a_{s}^{\dagger}\left(\omega^{\prime},-\omega, z\right) ;
\end{aligned}
$$


(ii) a pump equation,

$$
\begin{aligned}
\frac{\mathrm{d} a_{p}(\omega, z)}{\mathrm{d} z}= & \frac{i}{2} \frac{g_{0}}{\mathcal{A}_{p}} \int_{\mathscr{B}_{s}} \frac{\mathrm{d} \omega^{\prime}}{2 \pi}\left[\frac{\omega \omega^{\prime}\left(\omega-\omega^{\prime}\right)}{\Omega^{2} \Omega_{p}}\right]^{1 / 2} \\
& \times \exp \left[-i \Delta K\left(\omega, \omega^{\prime}\right) z\right] \frac{a_{s}\left(\omega^{\prime}, z\right) a_{s}\left(\omega-\omega^{\prime}, z\right)}{\left(\Delta_{p} / 2 \pi\right)^{1 / 2}} .
\end{aligned}
$$

These two equations are the spatial Langevin equations for our model of a DPA. The quantity

$$
\begin{aligned}
\Delta K\left(\omega^{\prime}, \omega\right) & \equiv \frac{\omega^{\prime} n_{0}}{c}-k(\omega)-k\left(\omega^{\prime}-\omega\right) \\
& =-\frac{\omega \Delta n(\omega)}{c}-\frac{\left(\omega^{\prime}-\omega\right) \Delta n\left(\omega^{\prime}-\omega\right)}{c}
\end{aligned}
$$

characterizes the phase mismatching between a pump frequency $\omega^{\prime}$ and signal frequencies $\omega$ and $\omega^{\prime}-\omega$.

The first term in the signal equation (2.37a) describes attenuation that is due to absorption, and the third term represents the fluctuations associated with absorption. The second and fourth terms are a consequence of the parametric nonlinearity; they are the same as the equivalent terms in the signal equation (2.35a) for an ideal medium, except for the presence of phase-mismatching factors. The characteristic size of the pump-fluctuation term is $1 / \mathcal{A}_{p}$ times the size of the primary nonlinear term. The pump equation $(2.37 \mathrm{~b})$ differs from the pump equation (2.35b) for an ideal medium because of a phase-mismatching factor.

The spatial Langevin equations (2.37) display clearly the classical-pump limit: $g_{0}=$ constant, $\mathcal{A}_{p} \rightarrow \infty$. In this limit the pump-fluctuation term in the signal equation goes away, and the pump is decoupled from the signal field.

It is instructive at this point to contrast our approach with the wave-packet formalism developed by Tucker and Walls, ${ }^{12}$ which has been applied to a DPA by Lane et al. ${ }^{19}$ In our approach, because we work in the temporal Fourier domain, frequency matching is enforced exactly. Just as in the usual classical analysis, phase mismatching appears as a mismatch $\Delta K\left(\omega^{\prime}, \omega\right)$ between wave numbers whose corresponding frequencies match exactly. Tucker and Walls idealize to an infinitely long medium so that wave-number matching is enforced exactly. In their formalism phase mismatching appears as a mismatch between frequencies whose corresponding wave numbers match exactly.

Before going further, we make a series of simplifications. We assume that the absorption coefficient is constant over the signal bandwidth, i.e., $\gamma(\omega)=\gamma$; we ignore the variation of the square-root-of-frequency factors in Eqs. (2.37); and we choose the pump phase to be $\phi_{p}=2 \phi=0$. With these assumptions, it is convenient to rewrite Eqs. (2.37) in terms of deviations of signal and pump frequencies from $\Omega$ and $\Omega_{p}$ :

$$
\begin{aligned}
\frac{\mathrm{d} a_{s}(\Omega+\epsilon, z)}{\mathrm{d} z}= & -1 / 2 \gamma a_{s}(\Omega+\epsilon, z) \\
& -g_{0} \exp [i \Delta k(\epsilon) z] a_{s}^{\dagger}(\Omega-\epsilon, z) \\
& +\gamma^{1 / 2} b_{s}(\Omega+\epsilon, z)+P(\epsilon, z),
\end{aligned}
$$

$$
\begin{aligned}
\frac{\mathrm{d} a_{p}\left(\Omega_{p}+\epsilon, z\right)}{\mathrm{d} z}= & \frac{i}{2} \frac{g_{0}}{\mathcal{A}_{p}} \int_{-\infty}^{\infty} \frac{\mathrm{d} \epsilon^{\prime}}{2 \pi} \exp \left[-i \Delta k\left(\epsilon^{\prime}, \epsilon^{\prime}-\epsilon\right) z\right] \\
& \times \frac{a_{s}\left(\Omega+\epsilon^{\prime}, z\right) a_{s}\left(\Omega+\epsilon-\epsilon^{\prime}, z\right)}{\left(\Delta_{p} / 2 \pi\right)^{1 / 2}} .
\end{aligned}
$$

Here the pump-fluctuation term is given by

$$
\begin{aligned}
P(\epsilon, z) \equiv & i \frac{g_{0}}{\mathcal{A}_{p}} \int_{-\infty}^{\infty} \frac{\mathrm{d} \epsilon^{\prime}}{2 \pi} \exp \left[i \Delta k\left(\epsilon, \epsilon^{\prime}\right) z\right] \frac{a_{p}\left(\Omega_{p}+\epsilon-\epsilon^{\prime}, z\right)}{\left(\Delta_{p} / 2 \pi\right)^{1 / 2}} \\
& \times a_{s}^{\dagger}\left(\Omega-\epsilon^{\prime}, z\right),
\end{aligned}
$$

and the phase mismatching is redefined in terms of

$$
\begin{aligned}
\Delta k\left(\epsilon, \epsilon^{\prime}\right) & \equiv \Delta K\left(\Omega_{p}+\epsilon-\epsilon^{\prime}, \Omega+\epsilon\right) \\
& =-\frac{(\Omega+\epsilon) \Delta n(\Omega+\epsilon)}{c}-\frac{\left(\Omega-\epsilon^{\prime}\right) \Delta n\left(\Omega-\epsilon^{\prime}\right)}{c}, \\
\Delta k(\epsilon) & \equiv \Delta k(\epsilon, \epsilon) \\
& =-\frac{(\Omega+\epsilon) \Delta n(\Omega+\epsilon)}{c}-\frac{(\Omega-\epsilon) \Delta n(\Omega-\epsilon)}{c}
\end{aligned}
$$

[cf. Eq. (2.4)]. In Eqs. (2.39) we formally extend the integration limits to $\pm \infty$, anticipating that in the calculations of Subsection 3.D the phase-mismatching factors provide a natural cutoff for the integrals.

We now introduce quadrature-phase amplitudes ${ }^{7,16,20}$ for the signal field, defined by

$$
\begin{aligned}
& \alpha_{1}(\epsilon, z) \equiv i / 2\left[a_{s}(\Omega+\epsilon, z)+a_{s}^{\dagger}(\Omega-\epsilon, z)\right], \\
& \alpha_{2}(\epsilon, z) \equiv-i / 2\left[a_{s}(\Omega+\epsilon, z)-a_{s}^{\dagger}(\Omega-\epsilon, z)\right] .
\end{aligned}
$$

The quadrature-phase amplitudes are the Fourier components of the quadrature phases of the signal field, defined with respect to frequency $\Omega$. Evaluated at $z=L$, they contain the spectral information about the squeezing produced by the DPA. They are defined here with respect to a phase such that when $\phi_{p}=0$ the $\alpha_{1}$ quadrature shows maximum squeezing near degeneracy. The frequency argument $\epsilon$ of a quadrature-phase amplitude is always assumed to be nonnegative.

Suppose that the output of the DPA is detected by a balanced homodyne detector. ${ }^{21,22}$ If the detector is ideal, the quadrature-phase amplitudes (multiplied by an appropriate factor) give the Fourier components of the differenced photocurrent at the output of the detector. ${ }^{7,23}$ Hence they provide the spectrum of the differenced photocurrent. Specifically, if the phase of the local oscillator powering the homodyne detector is chosen so as to display maximum squeezing for the phase-matched frequencies near degenera$\mathrm{cy}$, then $\alpha_{1}(\epsilon)$ gives the Fourier component at rf frequency $\epsilon$ of the differenced photocurrent.

We find it useful to introduce another set of quadraturephase amplitudes.

$$
\begin{array}{r}
\bar{\alpha}_{1}(\epsilon, z) \equiv{ }^{1 / 2}\left\{\exp [-i \Delta k(\epsilon) z / 2] a_{s}(\Omega+\epsilon, z)\right. \\
\left.+\exp [i \Delta k(\epsilon) z / 2] a_{s}^{\dagger}(\Omega-\epsilon, z)\right\},
\end{array}
$$




$$
\begin{gathered}
\bar{\alpha}_{2}(\epsilon, z) \equiv-i / 2\left\{\exp [-i \Delta k(\epsilon) z / 2] a_{s}(\Omega+\epsilon, z)\right. \\
\left.-\exp [i \Delta k(\epsilon) z / 2] a_{s}^{\dagger}(\Omega-\epsilon, z)\right\},
\end{gathered}
$$

which are related to the original quadrature-phase amplitudes by a frequency- and position-dependent rotation:

$$
\begin{aligned}
\bar{\alpha}_{1}(\epsilon, z)= & \alpha_{1}(\epsilon, z) \cos [\Delta k(\epsilon) z / 2] \\
& +\alpha_{2}(\epsilon, z) \sin [\Delta k(\epsilon) z / 2], \\
\bar{\alpha}_{2}(\epsilon, z)= & -\alpha_{1}(\epsilon, z) \sin [\Delta k(\epsilon) z / 2] \\
& +\alpha_{2}(\epsilon, z) \cos [\Delta k(\epsilon) z / 2] .
\end{aligned}
$$

Evaluated at $z=L$, these barred quadrature-phase amplitudes also contain the spectral information about the squeezing produced by the DPA but with some of the effects of phase mismatching removed. To detect these barred quadrature-phase amplitudes, one would have to vary the phase of the local oscillator in a homodyne detector as a function of rf frequency $\epsilon$.

We now put the signal equation (2.39a) into the form that we use in Section 3 by writing it in terms of the barred quadrature-phase amplitudes:

$$
\begin{aligned}
\frac{\mathrm{d} \bar{\alpha}_{1}(\epsilon, z)}{\mathrm{d} z}= & -\left(g_{0}+\frac{1}{2} \gamma\right) \bar{\alpha}_{1}(\epsilon, z)+\frac{1}{2} \Delta k(\epsilon) \bar{\alpha}_{2}(\epsilon, z) \\
& +\gamma^{1 / 2} \bar{\beta}_{1}(\epsilon, z)+\bar{P}_{1}(\epsilon, z) \\
\frac{\mathrm{d} \bar{\alpha}_{2}(\epsilon, z)}{\mathrm{d} z}= & +\left(g_{0}-\frac{1}{2} \gamma\right) \bar{\alpha}_{2}(\epsilon, z)-1 / 2 \Delta k(\epsilon) \bar{\alpha}_{1}(\epsilon, z) \\
& +\gamma^{1 / 2} \bar{\beta}_{2}(\epsilon, z)+\bar{P}_{2}(\epsilon, z) .
\end{aligned}
$$

Here

$$
\begin{aligned}
\bar{\beta}_{1}(\epsilon, z) \equiv & 1 / 2\left\{\exp [-i \Delta k(\epsilon) z / 2] b_{s}(\Omega+\epsilon, z)\right. \\
& \left.+\exp [i \Delta k(\epsilon) z / 2] b_{s}^{\dagger}(\Omega-\epsilon, z)\right\}, \\
\bar{\beta}_{2}(\epsilon, z) \equiv & -i / 2\left\{\exp [-i \Delta k(\epsilon) z / 2] b_{s}(\Omega+\epsilon, z)\right. \\
& \left.-\exp [i \Delta k(\epsilon) z / 2] b_{s}^{\dagger}(\Omega-\epsilon, z)\right\}
\end{aligned}
$$

are quadrature-phase amplitudes for the fluctuations associated with absorption, and the pump-fluctuation terms are defined by

$$
\begin{aligned}
\vec{P}_{1}(\epsilon, z) \equiv & 1 / 2\{\exp [-i \Delta k(\epsilon) z / 2] P(+\epsilon, z) \\
& \left.+\exp [i \Delta k(\epsilon) z / 2] P^{\dagger}(-\epsilon, z)\right\}, \\
\bar{P}_{2}(\epsilon, z) \equiv & -i / 2\{\exp [-i \Delta k(\epsilon) z / 2] P(+\epsilon, z) \\
& \left.-\exp [i \Delta k(\epsilon) z / 2] P^{\dagger}(-\epsilon, z)\right] .
\end{aligned}
$$

Equations (2.44) show that the primary effect of the parametric interaction is to deamplify (squeeze) the $\bar{\alpha}_{1}$ quadrature and to amplify the $\bar{\alpha}_{2}$ quadrature. This primary effect is degraded by absorption and phase mismatching.

\section{MODEL PREDICTIONS FOR SQUEEZING SPECTRA}

\section{A. Solution of Signal Equations}

We can write a formal Green-function solution of the signal equations (2.44) for the barred quadrature-phase amplitudes:

$$
\begin{aligned}
\bar{\alpha}_{m}(\epsilon, z)= & \sum_{n=1,2}\left\{\bar{G}_{m n}(\epsilon, z) \bar{\alpha}_{n}(\epsilon, 0)+\int_{0}^{z} \mathrm{~d} z^{\prime} \bar{G}_{m n}\left(\epsilon, z-z^{\prime}\right)\right. \\
& {\left.\left[\gamma^{1 / 2} \bar{\beta}_{n}\left(\epsilon, z^{\prime}\right)+\bar{P}_{n}\left(\epsilon, z^{\prime}\right)\right]\right\}, \quad m=1,2 . }
\end{aligned}
$$

Here the Green-function matrix is given by

$$
\begin{aligned}
& \bar{G}_{11}(\epsilon, z) \equiv e^{-\gamma z / 2} \frac{e^{-g z}-\mu^{2} e^{g z}}{1-\mu^{2}}, \\
& \bar{G}_{22}(\epsilon, z) \equiv e^{-\gamma z / 2} \frac{e^{g z}-\mu^{2} e^{-g z}}{1-\mu^{2}}, \\
& \bar{G}_{12}(\epsilon, z)=-\bar{G}_{21}(\epsilon, z)=\mu e^{-\gamma z / 2} \frac{e^{g z}-e^{-g z}}{1-\mu^{2}},
\end{aligned}
$$

where

$$
\begin{aligned}
& g=g(\epsilon) \equiv\left\{g_{0}{ }^{2}-[\Delta k(\epsilon) / 2]^{2}\right\}^{1 / 2}, \\
& \mu=\mu(\epsilon) \equiv \frac{\Delta k(\epsilon) / 2}{g_{0}+g(\epsilon)} .
\end{aligned}
$$

The Green-function matrix represents the familiar classical solution for a DPA with phase mismatching and absorption. Of course, only in the classical-pump limit, for which the pump-fluctuation terms $\bar{P}_{n}$ vanish, does Eq. (3.1) give an actual solution of the signal equations. In the presence of pump fluctuations, Eq. (3.1) is an integral equation, which can be used as the starting point for an iterative solution procedure.

If $\epsilon$ lies well within the phase-matched bandwidth $\Delta / 2 \pi$ [Eq. (2.8)], then $\Delta k(\epsilon) \rightarrow 0$. Thus the barred quadraturephase amplitudes become the same as the unbarred quadrature-phase amplitudes and, in addition, $g(\epsilon) \rightarrow g_{0}$ and $\mu(\epsilon) \rightarrow$ 0 , so that the Green-function matrix becomes diagonal, with the diagonal elements given by

$$
\bar{G}_{11}(\epsilon, z)=e^{-\gamma z / 2} e^{-g_{0} z}, \quad \bar{G}_{22}(\epsilon, z)=e^{-\gamma z / 2} e^{g_{0} z} .
$$

This is the classical solution for a phase-matched DPA with absorption; the parametric interaction deamplifies (squeezes) the $\alpha_{1}$ quadrature and amplifies the $\alpha_{2}$ quadrature.

\section{B. Squeezing Spectrum}

Our goal is to calculate squeezing spectra for the light generated by our model DPA. Spectral information about the squeezing produced by the DPA is contained in the spectraldensity matrix $7,16,20,23 S_{m n}(\epsilon)$ of the output quadraturephase amplitudes $\alpha_{m}(\epsilon, L)$. The spectral-density matrix arises from second-order noise moments of the quadraturephase amplitudes:

$$
\begin{array}{r}
\left\langle\Delta \alpha_{m}^{\dagger}\left(\epsilon^{\prime}, L\right) \Delta \alpha_{n}(\epsilon, L)\right\rangle_{\mathrm{sym}}=\pi S_{m n}(\epsilon) \delta\left(\epsilon-\epsilon^{\prime}\right), \\
m, n=1,2 .
\end{array}
$$


Here, for any operator $\mathcal{O}, \Delta \mathcal{O} \equiv \mathcal{O}-\langle\mathcal{O}\rangle$, and sym denotes a symmetrized product. A similar spectral-density matrix can be defined for the barred quadrature-phase amplitudes:

$$
\left\langle\Delta \bar{\alpha}_{m}^{\dagger}\left(\epsilon^{\prime}, L\right) \Delta \bar{\alpha}_{n}(\epsilon, L)\right\rangle_{\mathrm{sym}}=\pi \bar{S}_{m n}(\epsilon) \delta\left(\epsilon-\epsilon^{\prime}\right) .
$$

These two spectral-density matrices are related by

$$
\begin{aligned}
S_{11}= & \bar{S}_{11} \cos ^{2}(\Delta k L / 2)+\bar{S}_{22} \sin ^{2}(\Delta k L / 2) \\
& -\left(\bar{S}_{12}+\bar{S}_{21}\right) \cos (\Delta k L / 2) \sin (\Delta k L / 2), \\
S_{22}= & \bar{S}_{11} \sin ^{2}(\Delta k L / 2)+\bar{S}_{22} \cos ^{2}(\Delta k L / 2) \\
& +\left(\bar{S}_{12}+\bar{S}_{21}\right) \cos (\Delta k L / 2) \sin (\Delta k L / 2), \\
S_{12}= & S_{21}{ }^{*}=\left(\bar{S}_{11}-\bar{S}_{22}\right) \cos (\Delta k L / 2) \sin (\Delta k L / 2) \\
& +\bar{S}_{12} \cos ^{2}(\Delta k L / 2)-\bar{S}_{21} \sin ^{2}(\Delta k L / 2),
\end{aligned}
$$

where $\Delta k=\Delta k(\epsilon)$.

We are primarily interested in the spectrum of the squeezed quadrature, i.e., $S_{11}$ or $\bar{S}_{11}$. As noted in Subsection 2.C, $S_{11}(\epsilon)$ gives the spectrum of the differenced photocurrent in an ideal balanced homodyne detector, when the phase of the local oscillator is chosen so as to display maximum squeezing for the phase-matched frequencies near degeneracy. The spectrum $\bar{S}_{11}(\epsilon)$ would apply if one suitably varied the phase of the local oscillator as a function of $\mathrm{rf}$ frequency $\epsilon$.

We must also specify the spectra of the input signal field and the auxiliary field associated with absorption. We assume that both are in the vacuum state, which means that their first moments vanish and that their second moments are given by

$$
\begin{aligned}
\left\langle\alpha_{m}^{\dagger}\left(\epsilon^{\prime}, 0\right) \alpha_{n}(\epsilon, 0)\right\rangle_{\mathrm{sym}} & =\left\langle\bar{\alpha}_{m}^{\dagger}\left(\epsilon^{\prime}, 0\right) \bar{\alpha}_{n}(\epsilon, 0)\right\rangle_{\mathrm{sym}} \\
& =1 / 2 \pi \delta_{m n} \delta\left(\epsilon-\epsilon^{\prime}\right), \\
\left\langle\bar{\beta}_{m}^{\dagger}\left(\epsilon^{\prime}, z^{\prime}\right) \bar{\beta}_{n}(\epsilon, z)\right\rangle_{\mathrm{sym}} & =1 / 2 \pi \delta_{m n} \delta\left(\epsilon-\epsilon^{\prime}\right) \delta\left(z-z^{\prime}\right) .
\end{aligned}
$$

Recall also that we assume that the input pump field is in the vacuum state, aside from the strong excitation at frequency $\Omega_{p}$.

In the classical-pump limit $\left(\bar{P}_{m}=0\right)$, it is straightforward to calculate the output spectral-density matrix $\bar{S}_{m n}(\epsilon)$ in terms of the Green-function matrix:

$$
\begin{aligned}
\bar{S}_{m n}(\epsilon)= & \frac{1}{2} \sum_{p=1,2}\left[\bar{G}_{m p}{ }^{*}(\epsilon, L) \bar{G}_{n p}(\epsilon, L)\right. \\
& \left.+\gamma \int_{0}^{L} \mathrm{~d} z \bar{G}_{m p}{ }^{*}(\epsilon, L-z) \bar{G}_{n p}(\epsilon, L-z)\right] .
\end{aligned}
$$

The first term in this spectral-density matrix comes from the vacuum fluctuations in the input signal field, processed through the parametric interaction; this first term includes phase mismatching and attenuation that is due to absorption. The second term arises from the fluctuations associated with absorption. It is an integral over fluctuations injected at positions $z$ within the medium; after a fluctuation is injected at $z$, it is processed through the remainder of the nonlinear medium between $z$ and $L$.

If we assume further that $\epsilon$ lies well within the phase- matched bandwidth $\Delta / 2 \pi$, then $\bar{S}_{m n}(\epsilon)=S_{m n}(\epsilon)$ becomes diagonal, with the diagonal elements given by

$$
\begin{aligned}
& \bar{S}_{11}(\epsilon)=S_{11}(\epsilon)=\frac{1}{2} \frac{\gamma+2 g_{0} \exp \left[-\left(\gamma+2 g_{0}\right) L\right]}{\gamma+2 g_{0}}, \\
& \bar{S}_{22}(\epsilon)=S_{22}(\epsilon)=\frac{1}{2} \frac{\gamma-2 g_{0} \exp \left[-\left(\gamma-2 g_{0}\right) L\right]}{\gamma-2 g_{0}} .
\end{aligned}
$$

This is the familiar situation of squeezing competing with losses. In the absence of absorption, Eqs. (3.10) reduce further to the spectra for ideal squeezing:

$$
S_{11}(\epsilon)=1 / 2 e^{-2 g_{0} L}, \quad S_{22}(\epsilon)=1 / 2 e^{2 g_{0} L} .
$$

\section{Phase Mismatching}

The preceding analysis can be applied immediately to investigate the effect of phase mismatching on the squeezing spectrum. To isolate the phase-mismatching effect, we assume that there is no absorption $(\gamma=0)$ and that the pump is classical $\left(\vec{P}_{m}=0\right)$. Then the spectrum of the $\bar{\alpha}_{1}$ quadrature becomes

$$
\bar{S}_{11}(\epsilon)=1 / 2\left[\left|\bar{G}_{11}(\epsilon, L)\right|^{2}+\left|\bar{G}_{12}(\epsilon, L)\right|^{2}\right] .
$$

The significance of phase mismatching for $\bar{S}_{11}(\epsilon)$ is quantified by the dimensionless parameter

$$
\Delta k(\epsilon) / 2 g_{0}=-(p /|p|)\left(\epsilon / \epsilon_{2}\right)^{2}
$$

[cf. Eq. (2.7b)]. We assume that $\left|\Delta k(\epsilon) / 2 g_{0}\right| \ll 1$, i.e., $\epsilon \ll \epsilon_{2}$, and we then expand in $\left|\Delta k(\epsilon) / 2 g_{0}\right|$, keeping only the largest corrections to ideal squeezing. Under these circumstances, one sees that $g=g_{0}$ and

$$
\mu(\epsilon)=\Delta k(\epsilon) / 4 g_{0}=-1 / 2(p /|p|)\left(\epsilon / \epsilon_{2}\right)^{2} .
$$

When there is at least a moderate amount of squeezing, i.e., $g_{0} L$ is somewhat larger than 1 , the largest correction to ideal squeezing is the one that grows fastest with $g_{0} L$. This means that we can approximate

$$
\bar{G}_{11}(\epsilon, L)=e^{-g_{0} L}, \quad \bar{G}_{12}(\epsilon, L)=\mu(\epsilon) e^{g_{0} L},
$$

which leads to a squeezing spectrum

$$
\bar{S}_{11}(\epsilon)=1 / 2\left\{e^{-2 g_{0} L}+[\mu(\epsilon)]^{2} e^{2 g_{0} L}\right\}=1 / 2\left[e^{-2 g_{0} L}+1 / 4\left(\epsilon / \epsilon_{2}\right) 4 e^{2 g_{0} L}\right] .
$$

The dominant correction to ideal squeezing arises because phase mismatching feeds a fraction $|\mu(\epsilon)|$ of the amplified quadrature into the squeezed quadrature. In order to neglect phase mismatching and have ideal squeezing in the spectrum $\bar{S}_{11}(\epsilon)$, one requires

$$
|\mu(\epsilon)| e^{g_{0} L} \ll e^{-g_{0} L} \Rightarrow \epsilon \ll 2^{1 / 2} \epsilon_{2} e^{-g_{0} L} .
$$

To explore the effect of phase mismatching on the squeezing spectrum $S_{11}(\epsilon)$, one needs to consider an additional dimensionless parameter

$$
\Delta k(\epsilon) L=-(p /|p|)\left(\epsilon / \epsilon_{1}\right)^{2}
$$

[cf. Eq. (2.7a)]. Assuming that $|\Delta k(\epsilon) L| \ll 1$ and performing. a similar analysis to find the largest correction to ideal squeezing, one finds 


$$
S_{11}(\epsilon)=\frac{1}{2}\left\{e^{-2 g_{0} L}+[\mu(\epsilon)-1 / 2 \Delta k(\epsilon) L]^{2} e^{2 g_{0} L}\right\} .
$$

\section{Pump Quantum Fluctuations}

We turn now to an analysis of quantum fluctuations in the pump field. The starting point is the formal solution [Eq. (3.1)] for the squeezed quadrature $\bar{\alpha}_{1}(\epsilon, L)$. To simplify the analysis and to highlight the effect of pump fluctuations, we assume that there is no absorption $(\gamma=0)$, and we assume that $\epsilon$ lies well within the region of perfect phase matching $(\epsilon$ $\left.\ll \epsilon_{2} e^{-g_{0} L}\right)$. With these simplifications, we can write

$$
\bar{G}_{1 n}(\epsilon, z)=\delta_{1 n} e^{-g_{0} z},
$$

and the formal solution becomes

$$
\bar{\alpha}_{1}(\epsilon, L)=e^{-g_{0} L} \bar{\alpha}_{1}(\epsilon, 0)+\int_{0}^{L} \mathrm{~d} z \exp \left[-g_{0}(L-z)\right] \bar{P}_{1}(\epsilon, z) .
$$

One can solve Eq. (3.21) by an iterative procedure in which the fundamental expansion parameter is $1 / \mathcal{A}_{p} \ll 1$. The procedure is to evaluate $\bar{P}_{1}(\epsilon, z)$ to progressively higher orders in $1 / \mathcal{A}_{p}$ by plugging in progressively higher-order approximations to the signal and pump fields. Here we are interested only in the first-order correction to $\bar{\alpha}_{1}(\epsilon, L)$, so we can evaluate $\bar{P}_{1}(\epsilon, z)$ by using the zero-order solutions for the signal and pump fields.

Furthermore, we are interested in the dominant effect of pump fluctuations when there is at least a moderate amount of squeezing, i.e., $g_{0} L$ is somewhat larger than 1 . In this case
The next step is to substitute the zero-order solutions into Eq. (3.22). The zero-order solution for the pump is that the operators $a_{p}(\omega, z)=a_{p}(\omega)$ have no $z$ dependence (decoupling from the signal), and the zero-order solution for $\bar{\alpha}_{2}\left(\epsilon^{\prime}, z\right)$ is given by the first term in Eq. (3.1) with $\gamma=0$. This step taken, one then performs the integral over $z$ in Eq. (3.21). Before proceeding, however, the form [Eq. (3.22)] for $\bar{P}_{1}(\epsilon, z)$ permits a further dramatic simplification.

The phase-mismatching factors conspire in Eq. (3.22) to introduce new oscillating phase factors $\exp \left[i \Omega n^{\prime}\left(\epsilon^{\prime}-\epsilon\right) z / c\right]$ and $\exp \left[-i \Omega n^{\prime}\left(\epsilon^{\prime}+\epsilon\right) z / c\right]$. Once the integral over $z$ in Eq. (3.21) is done, these phase factors cut off the $\epsilon^{\prime}$ integral in Eq. (3.22). The first phase factor cuts off the integral when $\epsilon^{\prime}$ is sufficiently far from $\epsilon$ that the phase factor oscillates rapidly on the scale $g_{0}^{-1}$ set by the nonlinear gain. Similarly, the second phase factor cuts off the integral when $\epsilon^{\prime}$ is sufficiently far from $-\epsilon$. The frequency scale of these cutoffs is characterized by a frequency $\epsilon_{3}$, defined by

$$
\frac{\Omega\left|n^{\prime}\right| \epsilon_{3} / c}{2 g_{0}}=1 \Longrightarrow \epsilon_{3}=2 c g_{0} / \Omega\left|n^{\prime}\right| .
$$

Notice that $\epsilon_{3}$ is typically somewhat smaller than $\epsilon_{2}$ [Eq. (2.7b)]; indeed we assume that $\epsilon_{3} \ll \epsilon_{2} e^{-g_{0} L}$.

Since by assumption $\epsilon$ lies well within the region of perfect phase matching, and since $\epsilon^{\prime}$ in Eq. (3.22) is restricted to be within about a distance $\epsilon_{3}$ of $\epsilon$, we are entitled to use in Eq. (3.22) the perfectly phase-matched zero-order solution for $\bar{\alpha}_{2}\left(\epsilon^{\prime}, z\right)$, i.e., $\bar{\alpha}_{2}\left(\epsilon^{\prime}, z\right)=e^{g_{0} z} \bar{\alpha}_{2}\left(\epsilon^{\prime}, 0\right)$. Substituting the zeroorder solutions into Eq. (3.22), and performing the integral over $z$ in Eq. (3.21), one finds that

$$
\begin{aligned}
\bar{\alpha}_{1}(\epsilon, L)= & e^{-g_{0} L} \bar{\alpha}_{1}(\epsilon, 0)+\frac{e^{g_{0} L}}{4 \mathcal{A}_{p}} \int_{0}^{\infty} \frac{\mathrm{d} \epsilon^{\prime}}{2 \pi}\left\{\frac{\exp \left[i \Omega n^{\prime}\left(\epsilon^{\prime}-\epsilon\right) L / c\right]}{1+i \frac{\Omega n^{\prime}}{2 c g_{0}}\left(\epsilon^{\prime}-\epsilon\right)} \frac{a_{p}\left(\Omega_{p}+\epsilon-\epsilon^{\prime}\right)+a_{p}^{\dagger}\left(\Omega_{p}-\epsilon+\epsilon^{\prime}\right)}{\left(\Delta_{p} / 2 \pi\right)^{1 / 2}} \bar{\alpha}_{2}\left(\epsilon^{\prime}, 0\right)\right. \\
& \left.+\frac{\exp \left[-i \Omega n^{\prime}\left(\epsilon^{\prime}+\epsilon\right) L / c\right]}{1-i \frac{\Omega n^{\prime}}{2 c g_{0}}\left(\epsilon^{\prime}+\epsilon\right)} \frac{a_{p}\left(\Omega_{p}+\epsilon+\epsilon^{\prime}\right)+a_{p}^{\dagger}\left(\Omega_{p}-\epsilon-\epsilon^{\prime}\right)}{\left(\Delta_{p} / 2 \pi\right)^{1 / 2}} \bar{\alpha}_{2}^{\dagger}\left(\epsilon^{\prime}, 0\right)\right\}
\end{aligned}
$$

the dominant contribution to $\bar{P}_{1}$ comes from the amplified $\bar{\alpha}_{2}$ quadrature, so we can neglect the contribution to $\bar{P}_{1}$ from $\bar{\alpha}_{1}$.

With this final assumption in mind, we use Eqs. (2.46a), (2.39c), (2.40), and (2.42) to put $\vec{P}_{1}$ in the form

$$
\begin{aligned}
\bar{P}_{1}(\epsilon, z)= & 1 / 2 \frac{g_{0}}{\mathcal{A}_{p}} \int_{0}^{\infty} \frac{\mathrm{d} \epsilon^{\prime}}{2 \pi}\left\{\exp \left[i \Omega n^{\prime}\left(\epsilon^{\prime}-\epsilon\right) z / c\right]\right. \\
& \times \frac{a_{p}\left(\Omega_{p}+\epsilon-\epsilon^{\prime}, z\right)+a_{p}^{\dagger}\left(\Omega_{p}-\epsilon+\epsilon^{\prime}, z\right)}{\left(\Delta_{p} / 2 \pi\right)^{1 / 2}} \bar{\alpha}_{2}\left(\epsilon^{\prime}, z\right) \\
& +\exp \left[-i \Omega n^{\prime}\left(\epsilon^{\prime}+\epsilon\right) z / c\right] \\
& \left.\times \frac{a_{p}\left(\Omega_{p}+\epsilon+\epsilon^{\prime}, z\right)+a_{p}^{\dagger}\left(\Omega_{p}-\epsilon-\epsilon^{\prime}, z\right)}{\left(\Delta_{p} / 2 \pi\right)^{1 / 2}} \bar{\alpha}_{2}^{\dagger}\left(\epsilon^{\prime}, z\right)\right\},
\end{aligned}
$$

where we make explicit use of the Taylor expansion (2.5) for the index of refraction.
This equation is the basic result for the dominant effect of pump quantum fluctuations on the squeezed quadrature.

The form of Eq. (3.24) [or of Eq. (3.22)] confirms the. heuristic argument given in Subsection 2.B. The pump field can be decomposed into quadrature phases whose quadrature-phase amplitud'es are

$$
\begin{aligned}
& \alpha_{p 1}(\epsilon, z) \equiv \frac{1}{2}\left[a_{p}\left(\Omega_{p}+\epsilon, z\right)+a_{p}^{\dagger}\left(\Omega_{p}-\epsilon, z\right)\right], \\
& \alpha_{p 2}(\epsilon, z) \equiv-i / 2\left[a_{p}\left(\Omega_{p}+\epsilon, z\right)-a_{p}^{\dagger}\left(\Omega_{p}-\epsilon, z\right)\right] .
\end{aligned}
$$

When $\phi_{p}=0$, the strong mean pump field at frequency $\Omega_{p}$ has complex amplitude $i A_{p}$. Thus the $\alpha_{p 2}$ quadrature represents fluctuations that are in phase with the strong mean field, i.e., pump amplitude fluctuations; the $\alpha_{p 1}$ quadrature represents fluctuations that are $90^{\circ}$ out of phase with strong mean field, i.e., pump phase fluctuations. A glance at Eq. (3.24) shows that the dominant effect of pump quantum fluctuations comes from fluctuations in the $\alpha_{p 1}$ quadrature-phase fluctuations-which feed noise from the amplified signal quadrature into the squeezed signal quadrature. 
The characteristic size of this effect is given by the factor $e^{g_{0} L} / 4 \mathcal{A}_{p}$ in front of the integral in Eq. (3.24) [cf. Eq. (2.9)]. All that remains now is to use the integral to determine the pump bandwidth $\Delta_{p} / 2 \pi$.

Substituting Eq. (3.24) into the expression (3.6), one finds a flat squeezing spectrum

$$
\bar{S}_{11}(\epsilon)=\frac{1}{2}\left(\frac{e^{2 g_{0} L}+e^{+2 g_{0} L}}{16 \mathcal{A}_{p}^{2}}\right), \quad \epsilon \ll \epsilon_{2} e^{-g_{0} L},
$$

where the pump bandwidth is defined by

$$
\frac{\Delta_{p}}{2 \pi} \equiv \int_{-\infty}^{\infty} \frac{\mathrm{d} \epsilon}{2 \pi} \frac{1}{1+\left(\epsilon / \epsilon_{3}\right)^{2}}=\frac{1}{2} \epsilon_{3}=\frac{c g_{0}}{\Omega\left|n^{\prime}\right|}
$$

[cf. Eq. (2.17)]. As expected, the frequency $\epsilon_{3}$, which comes ultimately from phase mismatching, provides a cutoff for the pump bandwidth.

One further point deserves mention. We have calculated the first-order correction to $\vec{\alpha}_{1}(\epsilon, L)$ resulting from pump quantum fluctuations; this first-order correction goes as $1 / \mathcal{A}_{p}$. Squared in calculating the spectral density, it produces a correction to the spectrum that goes as $1 / \mathcal{A}_{p}{ }^{2}$. The second-order term in $\bar{\alpha}_{1}(\epsilon, L)$ due to pump fluctuations goes as $1 / \mathcal{A}_{p}{ }^{2}$. Multiplied by the zero-order solution $e^{-g_{0} L} \bar{\alpha}_{1}(\epsilon, 0)$ in forming the spectral density, this second-order term also yields a correction to the spectrum that goes as $1 / \mathcal{A}_{p}{ }^{2}$. Why have we ignored this correction when it is formally of the same size as the effect that we have calculated? Because one can convince oneself, either by tedious analysis or by clever insight; that the correction to the spectrum that is due to the second-order term does not grow so fast as $e^{2 g_{0} L}$. Hence, the correction that we have calculated is the dominant effect when there is at least a moderate amount of squeezing.

\section{ACKNOWLEDGMENTS}

This research was supported in part by Caltech's Program in Advanced Technologies, sponsored by Aerojet General, General Motors, GTE, and TRW.

Note added in proof. A recent paper by Potasek and Yurke $^{24}$ on four-wave mixing in an optical fiber obtained results that are formally identical to Eqs. (3.2) and (3.9) in the absence of absorption $(\gamma=0)$. It prompted us to realize that Eqs. (3.16) and (3.19) overstate the deleterious effects of phase mismatching. A frequency-dependent rotation of the barred quadrature-phase amplitudes $\bar{\alpha}_{1}(\epsilon, L)$ and $\bar{\alpha}_{2}(\epsilon$, $L)$ [similar to the rotation of $\alpha_{1}(\epsilon, L)$ and $\alpha_{2}(\epsilon, L)$ in Eqs. (2.43)] by an appropriate angle $\theta(\epsilon, L)$ diagonalizes the resulting spectral-density matrix $\tilde{S}_{m n}(\epsilon)$. This choice of $\theta(\epsilon, L)$ minimizes (maximizes) $\tilde{S}_{11}(\epsilon)\left[\tilde{S}_{22}(\epsilon)\right]$. Keeping only the dominant correction to ideal squeezing for $g_{0} L$ somewhat larger than 1 and for $\epsilon \ll \epsilon_{2}$, one finds that

$$
\tilde{S}_{11}(\epsilon)=1 / 2 e^{-2 g_{0} L}\left\{1+4[\mu(\epsilon)]^{2} g_{0} L\right\}=1 / 2 e^{-2 g_{0} L}\left[1+\left(\epsilon / \epsilon_{2}\right)^{4} g_{0} L\right] .
$$

The dominant correction arises from the reduction in the nonlinear gain due to phase mismatching [Eq. (3.3a)]. In order to ignore phase mismatching and achieve ideal squeezing in the spectrum $\tilde{S}_{11}(\epsilon)$, one requires that

$$
2|\mu(\epsilon)|\left(g_{0} L\right)^{1 / 2} \ll 1 \Longrightarrow \epsilon \ll \epsilon_{2}\left(g_{0} L\right)^{-1 / 4}
$$

Relation (3.29) imposes a much less stringent restriction on the radio frequency $\epsilon$ than does expression (3.17).

\section{REFERENCES}

1. H. Takahashi, Adv. Commun. Syst. 1, 227 (1965), especially Sec. XI.

2. E. Y. C. Lu, Lett. Nuovo Cimento 3, 585 (1972).

3. D. Stoler, Phys. Rev. Lett. 33, 1397 (1974).

4. R. G. Smith, in Laser Handbook, F. T. Arecchi and E. O. SchulzDubois, eds. (North-Holland, Amsterdam, 1972), Vol. I, p. 837.

5. L.-A. Wu, H. J. Kimble, J. L. Hall, and H. Wu, Phys. Rev. Lett. 57,2520 (1986).

6. B. Yurke, Phys. Rev. A 29, 408 (1984).

7. B. Yurke, Phys. Rev. A 32, 300 (1985).

8. M. J. Collett and C. W. Gardiner, Phys. Rev. A 30, 1386 (1984).

9. C. W. Gardiner and C. M. Savage, Opt. Commun. 50, 173 (1984).

10. M. J. Collett and D. F. Walls, Phys. Rev. A 32, 2887 (1985).

11. Y. R. Shen, Phys. Rev. 155, 921 (1967).

12. J. Tucker and D. F. Walls, Phys. Rev. 178, 2036 (1969).

13. M. Hillery and L. D. Mlodinow, Phys. Rev. A 30, 1860 (1984).

14. M. Hillery and M. S. Zubairy, Phys. Rev. A 29, 1275 (1984).

15. C. M. Caves, Phys. Rev. D 23, 1693 (1981).

16. C. M. Caves and B. L. Schumaker, Phys. Rev. A 31, 3068 (1985).

17. K. Wódkiewicz and M. S. Zubairy, Phys. Rev. A 27, 2003 (1983).

18. G. Scharf and D. F. Walls, Opt. Commun. 50, 245 (1984).

19. A. Lane, P. Tombesi, H. J. Carmichael, and D. F. Walls, Opt. Commun. 48, 155 (1983).

20. C. M. Caves, Phys. Rev. D 26, 1817 (1982).

21. H. P. Yuen and V. W. S. Chan, Opt. Lett. 8, 177 (1983).

22. B. L. Schumaker, Opt. Lett. 9, 189 (1984).

23. C. M. Caves and B. L. Schumaker, in Quantum Optics IV, J. D. Harvey and D. F. Walls, eds. (Springer, Berlin, 1986), p. 20.

24. M. J. Potasek and B. Yurke, Phys. Rev. A 35, 3974 (1987).

\section{Carlton M. Caves}

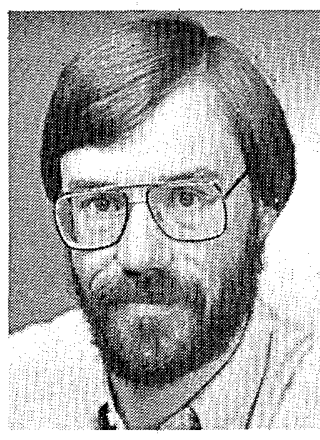

Carlton M. Caves was born in Muskogee, Oklahoma, on October 24, 1950. He received the B.A. degree in physics and mathematics from Rice University in 1972. After receiving the Ph.D. degree in physics from Caltech in 1979 , he was a research fellow in physics at Caltech before assuming his current position as a senior research fellow in theoretical physics. His research interests include gravitational-wave detection, quantum limitations on high-precision measurements, and quantum optics.

\section{David D. Crouch}

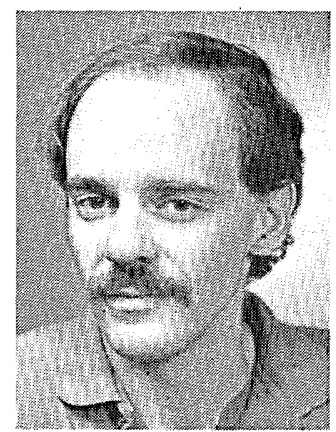

David D. Crouch was born in Petaluma, California, on December 2, 1960. He received the B.S. degree from the University of California, Davis, in 1983 and the M.S. degree from Caltech in 1985, both in electrical engineering. He is currently working toward the Ph.D. degree in applied physics at Caltech. Mr. Crouch is a member of the Optical Society of America, the Society of Photo-Optical Instrumentation Engineers, and Tau Beta Pi. 